\title{
Secondary osteosarcoma arising from osteochondroma following autologous stem cell transplantation with total-body irradiation for neuroblastoma: A case report
}

\author{
HIROYUKI KAWASHIMA ${ }^{1}$, AKIRA OGOSE ${ }^{1}$, TETSUO HOTTA ${ }^{1}$, CHIHAYA IMAI $^{2}$, \\ MASAHARU IMAMURA ${ }^{2}$ and NAOTO ENDO ${ }^{1}$ \\ ${ }^{1}$ Division of Orthopedic Surgery, Department of Regenerative and Transplant Medicine; \\ ${ }^{2}$ Division of Pediatrics, Department of Homeostatic Regulation and Development, \\ Niigata University Graduate School of Medical and Dental Sciences, Niigata 951-8510, Japan
}

Received September 18, 2014; Accepted May 8, 2015

DOI: 10.3892/ol.2015.3257

\begin{abstract}
The present study reports the first case of malignant transformation to osteosarcoma arising from osteochondroma following childhood total-body irradiation (TBI). The association between TBI and later development of osteochondroma is well-known; however, malignant degeneration arising from radiation-induced osteochondroma is rare. The current study describes the case of a 17-year-old boy with osteosarcoma arising from osteochondroma of the left distal humerus, which developed following TBI. TBI was administered as part of a conditioning regimen received prior to autologous peripheral hematopoietic stem cell transplantation (HSCT) at the age of 6 years, following an initial diagnosis of neuroblastoma at the age of 5 years. The patient subsequently underwent preoperative chemotherapy followed by wide local excision and reconstruction with an extracorporeally irradiated autograft. Postoperative chemotherapy was administered, and the patient demonstrated no clinical or radiographic evidence of recurrence after 40 months of follow-up. To the best of our knowledge, this is only the second reported case of malignant degeneration of osteochondroma following childhood TBI, and the first reported case of transformation to osteosarcoma. The current case highlights the importance of close observation for secondary malignancies in this patient population.
\end{abstract}

Correspondence to: Dr Hiroyuki Kawashima, Division of Orthopedic Surgery, Department of Regenerative and Transplant Medicine, Niigata University Graduate School of Medical and Dental Sciences, 1-757 Asahimachi-dori, Chūō, Niigata 951-8510, Japan

E-mail: inskawa@med.niigata-u.ac.jp

Key words: osteosarcoma, osteochondroma, total-body irradiation, secondary malignancy

\section{Introduction}

Hematopoietic stem cell transplantation (HSCT) is a successful treatment strategy for children with potentially fatal diseases, such as hematological malignancies, severe aplastic anemia, inherited diseases or neuroblastoma (1). Prior to undergoing HSCT, high-dose chemotherapy and total-body irradiation (TBI) are administered in an attempt to eradicate residual malignant cells and cure the patient; however, the high-dose chemotherapy and radiotherapy may increase the risk of adverse effects in almost every organ or system in the body. In particular, the major skeletal complications observed in TBI survivors are osteoporosis, avascular necrosis and benign or malignant bone tumors.

Osteochondroma is a benign cartilage-capped tumor that predominantly develops at the juxtaepiphyseal region of the long bones. There have been few reports of osteochondroma as a secondary bone tumor following the treatment of childhood neuroblastoma (2-4); however, there are considerable experimental and clinical data linking osteochondroma to localized high-dose and TBI (5). The rate of malignant transformation in primary osteochondroma, principally into chondrosarcoma, is estimated to be $1-5 \%$ (6), and transformation into osteosarcoma is rare. The simultaneous occurrence of osteosarcoma and osteochondroma following treatment of neuroblastoma with chemotherapy, radiotherapy and autologous peripheral HSCT rescue has been reported previously (7). However, the current literature indicates a low incidence of malignant degeneration in radiation-induced osteochondroma (8-10) and only one case of chondrosarcoma arising within radiation-induced osteochondroma following childhood TBI has been reported thus far (11).

Herein, the present study reports a case of osteosarcoma arising from osteochondroma 11 years after the patient underwent surgery, local irradiation, chemotherapy, TBI and HSCT for neuroblastoma. Due to the radiographic characteristics and localization of the tumor, it is postulated that the osteosarcoma arose from osteochondroma and developed following TBI. To the best of our knowledge, this is the first reported case of its type. Written informed consent was obtained from the patient's family. 


\section{Case report}

A 5-year-old boy was admitted to Niigata University Hospital (Niigata, Japan) in January 1998 with a 2-week history of fever, cough, fatigue, headache and abdominal pain. On physical examination, a gross abdominal mass was palpable. Abdominal computed tomography (CT) demonstrated a retroperitoneal mass located adjacent to the left kidney and multiple enlarged lymph nodes along the aorta. The patient underwent an open biopsy, and pathological examination revealed a diagnosis of neuroblastoma, revealing undifferentiated unfavorable histology, DNA diploidy and MYCN oncogene amplification (12 copies). Multiple metastases to the occipital bone, left humerus, left tibia and bone marrow were indicated upon metaiodobenzylguanidine ( $\left.{ }^{123} \mathrm{I}-\mathrm{MIBG}\right)$ scanning. In addition, urine vanillylmandelic acid (VMA), homovanillic acid (HVA) and serum neuron-specific enolase (NSE) levels were elevated to $86.8 \mathrm{mg} / \mathrm{g}$ creatinine $(\mathrm{Cr}$; normal range VMA, 5.8-19.1 mg/g Cr), $103.7 \mathrm{mg} / \mathrm{g} \mathrm{Cr}$ (normal range HVA, 9.4-23.4 $\mathrm{mg} / \mathrm{g} \mathrm{Cr}$ ) and $>200 \mathrm{ng} / \mathrm{ml}$ (normal range NSE, $<16.3 \mathrm{ng} / \mathrm{ml}$ ), respectively. On the basis of these findings, the patient was diagnosed with stage IV neuroblastoma and considered to belong to the high-risk group, according to the International Neuroblastoma Staging System (12).

Following the diagnosis of neuroblastoma, the patient was treated according to the nationwide standard protocol established by the Japan Study Group for Advanced Neuroblastoma (13). Induction therapy with one cycle of regimen A1, consisting of cyclophosphamide (CPA; 1,200 mg/m²; day 1), etoposide (100 mg/m²; days $1-5)$, tetrahydropyranyl-Adriamycin ${ }^{\circledR}$ (THP-ADM; $40 \mathrm{mg} / \mathrm{m}^{2}$; day 3) and cisplatin (90 mg/m²; day 5), and three cycles of regimen A3, consisting of CPA $\left(1,200 \mathrm{mg} / \mathrm{m}^{2}\right.$; days 1-2), etoposide (100 mg/m²; days 1-5), THP-ADM (40 mg/m² day 3) and cisplatin ( $25 \mathrm{mg} / \mathrm{m}^{2}$, days 15$)$, was repeated every 4 weeks. Following completion of these 4 cycles, residual metastatic lesions were identified by performing a CT scan. Therefore, ifosfamide-based salvage chemotherapy was immediately undertaken, including one cycle of ifosfamide, carboplatin (IC) and etoposide (ICE), containing $4.5 \mathrm{~g} / \mathrm{m}^{2}$ ifosfamide (day 2), $385 \mathrm{mg} / \mathrm{m}^{2}$ carboplatin (day 2) and $100 \mathrm{mg} / \mathrm{m}^{2}$ etoposide (days 1-5), and two cycles of IC, containing $6 \mathrm{mg} / \mathrm{m}^{2}$ ifosfamide and $500 \mathrm{mg} / \mathrm{m}^{2}$ carboplatin, followed by administration of high-dose melphalan $\left(70 \mathrm{mg} / \mathrm{m}^{2}\right.$ on consecutive days) with concomitant preoperative autologous peripheral HSCT. Following induction chemotherapy, tumor markers were still not normalized (VMA, $47.8 \mathrm{mg} / \mathrm{g} \mathrm{Cr}$; HVA, $31.2 \mathrm{mg} / \mathrm{g} \mathrm{Cr}$; NSE, $9.7 \mathrm{ng} / \mathrm{ml}$ ) and an MIBG hotspot indicated that a small lesion remained in the left tibia. Thus, gross total resection to remove the entire macroscopic tumor from the primary site, including the regional lymph nodes, and combined resection of the left kidney were performed. This was followed by 12 Gy of intraoperative radiation therapy of the tumor bed and para-aortic lymph nodes. One cycle of regimen $\mathrm{C}$ chemotherapy, containing cyclophosphamide $\left(1,500 \mathrm{mg} / \mathrm{m}^{2}\right.$; day 1) and dacarbazine $\left(250 \mathrm{mg} / \mathrm{m}^{2}\right.$; days $\left.1-5\right)$, was administered postoperatively. Peripheral blood stem cells were collected by apheresis using positive cluster of differentiation $34^{+}$selection and the presence of tumor cells was detected by immunofluorescence. High-dose consolidation therapy was then administered, consisting of thiotepa $\left(200 \mathrm{mg} / \mathrm{m}^{2}\right.$ intravenously, twice daily for 2 days; total dose, $800 \mathrm{mg} / \mathrm{m}^{2}$ ) and $12 \mathrm{~Gy}$ of fractionated
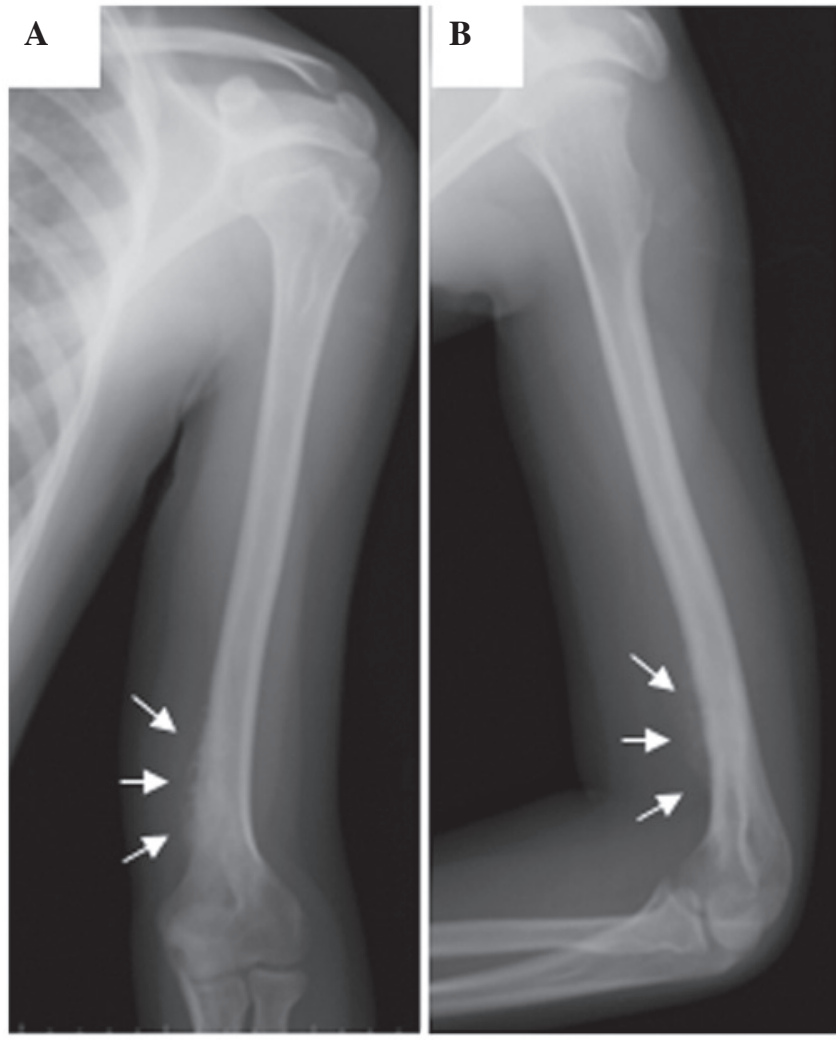

Figure 1. (A) Anteroposterior and (B) lateral radiographs captured 11 years after total-body irradiation indicating a poorly defined, broad surface-based lesion arising from the distal shaft of the humerus, with a spiculated and aggressive periosteal reaction perpendicular to the long axis of the humerus. The normal cortex is continuous with the lesion.

TBI, followed by autologous peripheral HSC rescue. TBI was delivered using 12 Gy radiation in 6 doses of 2-Gy fractions twice-daily for 3 consecutive days. Tumor markers, including serum NSE, and urinary HVA and VMA became normalized and were maintained within the normal ranges immediately after completion of the radiotherapy.

The patient developed multiple osteochondromas $\sim 6$ years after being treated for neuroblastoma in February 2004, despite having no clinically detectable bony masses prior to the neuroblastoma treatment and no family history of hereditary multiple exostoses. At this time, the patient did not have any experience pain or discomfort and did not receive any treatment following this diagnosis. At the age of 17 years in August 2009, 11 years after treatment for neuroblastoma, the patient presented to our clinic with a 1-week history of intermittent stabbing pain in the upper left arm. Physical examination revealed swelling along the distal aspect of the upper left arm, and radiography of the left elbow identified a periosteal reaction in the distal humeral shaft and an associated medial soft-tissue mass (Fig. 1). ${ }^{99} \mathrm{~m}$ Tc-MDP radionuclide bone scanning revealed a single focus of increased activity in the region of the left distal humerus. Subsequent magnetic resonance imaging of the tumor demonstrated an absence of medullary involvement, an irregular cortical surface and a soft-tissue mass (Fig. 2). The subperiosteal location of the tumor was clear and the imaging features were typical of osteosarcoma arising from osteochondroma. The remainder of the metastatic work-up, 

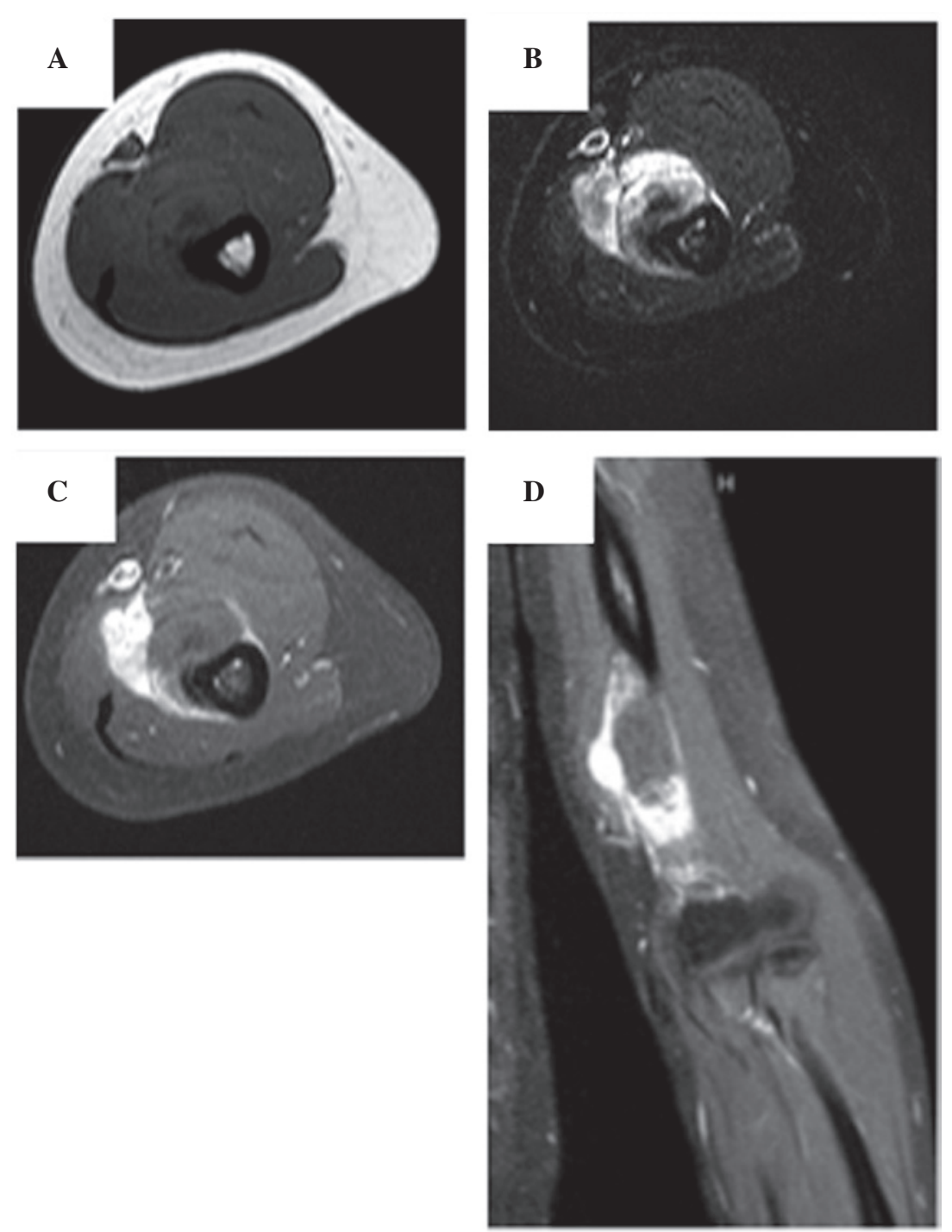

Figure 2. (A) Axial T1-weighted magnetic resonance image revealing a circumferential soft tissue mass around the distal shaft of the left humerus, with similar signal intensity to muscle. (B) Fat-saturated T2-weighted magnetic resonance image indicating that the soft tissue component around the surface of the bone has low signal intensity due to dense matrix mineralization. The more peripheral component is hyperintense, reflecting the chondroblastic content of this tumor. (C) Axial and (D) sagittal post-contrast fat-suppressed T1-weighted magnetic resonance images demonstrating enhancement of the peripheral and a less densely mineralized component of the soft tissue mass.

including chest radiography, whole-body CT and 24-h monitoring of urinary VMA, dopamine and catecholamine levels, revealed no abnormalities. In addition, there was no family history of malignancy. Fine-needle aspiration cytology revealed oval- to spindle-shaped cells, a number of which had a high nucleocytoplasmic ratio with hyperchromatic nuclei, irregular nuclear border and prominent nucleoli. Additionally, osteoblasts with pronounced nuclear atypia were observed and diagnosed as osteosarcoma. Due to the heminephrectomy and chemotherapy previously undergone for the treatment of neuroblastoma, renal and liver dysfunction, as well as prolonged myelosuppression, were observed following two cycles of preoperative chemotherapy, comprising cisplatin $\left(100 \mathrm{mg} / \mathrm{m}^{2}\right.$; day 1$)$ and doxorubicin (25 $\mathrm{mg} / \mathrm{m}^{2}$; days 1-2). Furthermore, severe neuralgic pain in the jaw and abdomen was precipitated by an additional cycle of single agent chemotherapy comprising $2 \mathrm{mg}$ vincristine.

One month after completion of three cycles of preoperative chemotherapy, the patient underwent wide local excision of the tumor followed by reconstruction with an extracorporeally irradiated autograft combined with an iliac bone graft (Fig. 3). All bone segments were administered with a single fraction of $70 \mathrm{~Gy}$ of radiation. Following resection, the patient received postoperative chemotherapy, including nine cycles of high-dose methotrexate $\left(7 \mathrm{~g} / \mathrm{m}^{2}\right)$, two cycles of ifosfamide (3 mg/m $\mathrm{m}^{2}$; days $\left.1-2\right)$ and doxorubicin $\left(30 \mathrm{mg} / \mathrm{m}^{2}\right.$; days 1-2), one cycle of ifosfamide $\left(1 \mathrm{~g} / \mathrm{m}^{2}\right.$; days $\left.1-4\right), 4.5 \mathrm{mg}$ vindesine (day 1) and carboplatin $\left(100 \mathrm{mg} / \mathrm{m}^{2}\right.$; days $\left.1-4\right)$, and one cycle of IC, containing $1 \mathrm{mg} / \mathrm{m}^{2}$ ifosfamide (days 1-2) and $100 \mathrm{mg} / \mathrm{m}^{2}$ carboplatin (days 1-2). Doses of nephrotoxic cytostatic agents were reduced due to renal impairment. Postoperatively, immobilization with long arm brace was continued until there was radiographic evidence of union. A final pathological examination identified disease-free margins and $<50 \%$ necrosis of high-grade osteosarcoma (Fig. 4). After 40 months of follow-up, no clinical or radiographic evidence of recurrence or metastasis were observed in the current patient. 


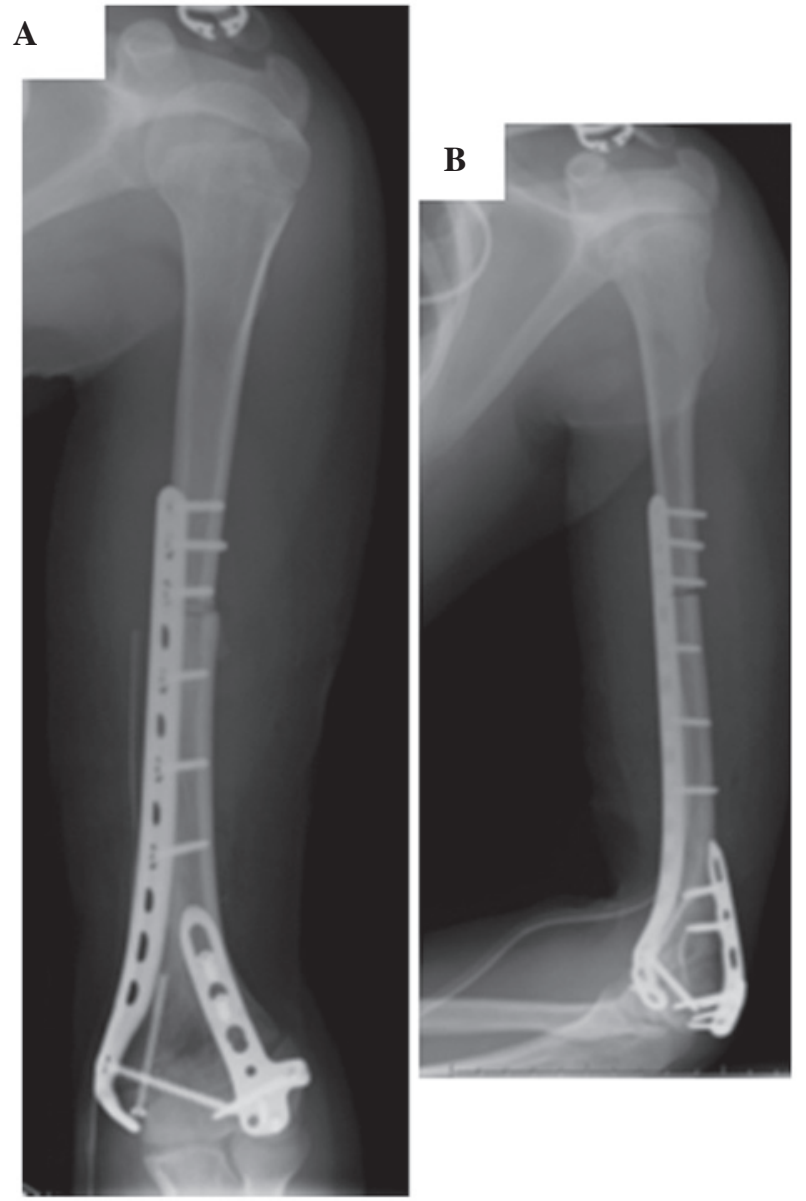

Figure 3. (A) Anteroposterior and (B) lateral radiographs captured immediately postoperatively demonstrating reimplantation of $11 \mathrm{~cm}$ irradiated bone and double-plate fixation of the graft.

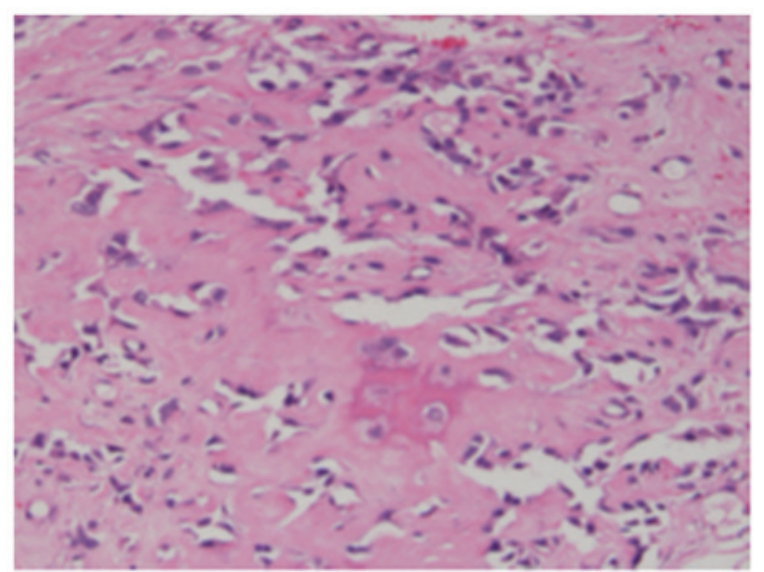

Figure 4. High-power photomicrograph revealing atypical cells with small hyperchromatic nuclei and inconspicuous eosinophilic cytoplasm, and osteoid formation by tumor cells (hematoxylin and eosin staining). Magnification, $\mathrm{x} 400$

\section{Discussion}

Advances in the diagnosis and management of pediatric cancer have led to increasing long-term survival rates (14). The initial follow-up of off-treatment patients is aimed at detecting relapse and treatment-associated morbidity (15).
Over time, surveillance includes monitoring for late effects of therapy, including secondary malignancies, the risk of which is higher in childhood cancer survivors compared with the general population $(16,17)$. A study of 14,358 childhood cancer survivors by the Childhood Cancer Survivor Study cohort identified an increased risk of second malignant neoplasm (SMN) among all primary childhood cancer diagnoses. When compared with the general population, the overall standardized incidence ratio of developing SMN was 6.4, with an estimated 30 -year cumulative incidence rate of $9.3 \%$ (17). The use of high-dose chemotherapy to eradicate disease in these aggressive pediatric malignancies, particularly alkylating agents, anthracyclines, and epipodophyllotoxins, has been shown to increase the risk of developing SMN (18). Furthermore, radiation and autologous HSCT are also known to increase the risk of SMN in children. In one of the largest studies to date, the Center for International Blood and Marrow Transplant Research assembled a cohort of 1,487 pediatric autologous transplant recipients with a median age of 8 years (range, 1-21 years). SMN was reported in 35 patients [AML/myelodysplastic syndrome (MDS), $n=13$; solid tumor, $n=20$; subtype unknown, $n=2$ ] and the overall cumulative incidence of SMN 10 years after autologous HSCT was $2.60 \%$ (AML/MDS, $1.06 \%$; solid tumor, $1.30 \%$ ). When compared with an age- and gender-matched general population, autologous HSCT recipients exhibited a 24-fold greater risk of developing SMN (95\% confidence interval, 16.0-33.0) (19).

Undergoing autologous HSCT also increases the risk of developing osteochondroma. Nine Italian HSCT centers of the Italian Association of Pediatric Hematology and Oncology assembled a cohort of 1,632 pediatric transplant recipients; among them, a total of 27 cases of osteochondroma (1.6\%) were reported. Analysis of cumulative risk stratified by various risk factors revealed that male gender, autologous HSCT, age of $<3$ years at time of HSCT and TBI significantly affected the risk of osteochondroma. However, malignant degeneration of osteochondroma was not noted (20).

Malignant transformation of osteochondroma into low-grade chondrosarcoma predominantly occurs in the cartilaginous cap. This phenomenon is considered to occur stepwise, as follows: 'Osteochondroma giving rise to peripheral low-grade chondrosarcoma that in turn dedifferentiates into a high-grade sarcoma that may appear as fibrosarcoma, malignant fibrous histiocytoma, and osteosarcoma' (21). Low-grade chondrosarcomas typically grow in the periphery, enlarging the cartilaginous cap and, thus, producing clinical indicators that can be identified on magnetic resonance imaging (22-24). Certain cases of osteosarcoma secondary to osteochondroma corroborate the stepwise theory and are composed of numerous types of neoplastic tissue, such as normal osteochondroma, low-grade chondrosarcoma and higher-grade osteosarcoma with a chondral component, as described by Nojima et al (25). Other cases develop from the spongy bone of the stalk and have no association with the cartilaginous cap. In this type of secondary osteosarcoma, no thickening of the cap is observed and a neoplastic cartilaginous component is not present $(22,26,27)$.

To the best of our knowledge, one previous report of simultaneous occurrence of osteosarcoma and osteochondroma following treatment of neuroblastoma with chemotherapy, 
radiotherapy and BMT exists in the literature. The study reported the case of an 11-year-old patient who presented with osteosarcoma of the fourth right rib and osteochondroma of the left scapula 9.5 years after receiving radiation, chemotherapy, TBI and BMT for abdominal neuroblastoma. However, the study did not report malignant transformation of osteochondroma (7). Additionally, one study reported the case of a 34-year-old man with chondrosarcoma arising from radiation-induced osteochondroma of the left posterior eighth rib. The osteochondroma developed following TBI received as part of a conditioning regimen prior to undergoing BMT at the age of 8 years (11).

The development of bone tumors in the current patient may be attributed to more than one factor. For example, it may be associated with the previous chemotherapy and radiotherapy that were administered to treat the neuroblastoma 10 years earlier. The present case illustrates the difficulty of assigning a single factor to secondary tumors in a patient with cancer, particularly when the patient has been treated with numerous therapeutic modalities.

In conclusion, the current study presents the first reported case of malignant transformation of osteochondroma into osteosarcoma associated with TBI. Treatment with TBI prior to HSCT, particularly in younger patients, results in a large number of epiphyses at risk of injury and at an increased risk of malignant degeneration, compared with osteochondroma arising in the absence of radiotherapy. Considering that the average age of malignant degeneration appears to be lower in this group of patients, surveillance may have a role in clinical management in order to avoid late complications of malignant degeneration and to improve opportunities for curative treatment when this rare condition occurs.

\section{References}

1. Faraci M, Barra S, Cohen A, et al: Very late nonfatal consequences of fractionated TBI in children undergoing bone marrow transplant. Int J Radiat Oncol Biol Phys 63: 1568-1575, 2005.

2. Paulino AC and Fowler BZ: Secondary neoplasms after radiotherapy for a childhood solid tumor. Pediatr Hematol Oncol 22: 89-101, 2005.

3. Bordigoni P, Turello R, Clement L, et al: Osteochondroma after pediatric hematopoietic stem cell transplantation: Report of eight cases. Bone Marrow Transplant 29: 611-614, 2002.

4. Paulino AC, Mayr NA, Simon JH, and Buatti JM: Locoregional control in infants with neuroblastoma: Role of radiation therapy and late toxicity. Int J Radiat Oncol Biol Phys 52: 1025-1031, 2002.

5. Marcovici PA, Berdon WE and Liebling MS: Osteochondromas and growth retardation secondary to externally or internally administered radiation in childhood. Pediatr Radiol 37: 301-304, 2007.

6. Bovée JVMG, Heymann D and Wuyts W: Osteochondroma. In: WHO Classification of Tumours of Soft Tissue and Bone. Fletcher DM, Bridge JA, Hogendoorn PCW and Mertens F (eds). IARC, Lyon, pp250-251, 2013.
7. Poustchi-Amin M, Leonidas JC and Elkowitz SS: Simultaneous occurrence of osteosarcoma and osteochondroma following treatment of neuroblastoma with chemotherapy, radiotherapy, and bone marrow transplantation. Pediatr Radiol 26: 155-157, 1996.

8. Perez CA, Vietti T, Ackerman LV, Eagleton MD and Powers WE: Tumors of the sympathetic nervous system in children. An appraisal of treatment and results. Radiology 88: 750-760, 1967.

9. Breslow NE, Takashima JR, Whitton JA, et al: Second malignant neoplasms following treatment for Wilm's tumor: A report from the National Wilms' Tumor Study Group. J Clin Oncol 13: 1851-1859, 1995.

10. Mahboubi S, Dormans JP and D'Angio G: Malignant degeneration of radiation-induced osteochondroma. Skeletal Radiol 26: 195-198, 1997.

11. Nagata S, Shen RK, Laack NN, et al: Chondrosarcoma arising within a radiation-induced osteochondroma several years following childhood total body irradiation: Case report. Skeletal Radiol 42: 1173-1177, 2013.

12. Brodeur GM, Pritchard J, Berthold F, et al: Revisions of the international criteria for neuroblastoma diagnosis, staging, and response to treatment. J Clin Oncol 11: 1466-1477, 1993.

13. Suita S, Tajiri T, Sera Y, et al: Improved survival for patients with advanced neuroblastoma after high-dose combined chemotherapy based in part on N-myc amplification. J Pediatr Surg 35: 1737-1741, 2000.

14. Mariotto AB, Rowland JH, Yabroff KR, et al: Long-term survivors of childhood cancers in the United States. Cancer Epidemiol Biomarkers Prev 18: 1033-1040, 2009.

15. Goldsby RE, Taggart DR and Ablin AR: Surviving childhood cancer: The impact on life. Paediatr Drugs 8: 71-84, 2006.

16. Friedman DL, Whitton J, Leisenring W, et al: Subsequent neoplasms in 5-year survivors of childhood cancer: The Childhood Cancer Survivor Study. J Natl Cancer Inst 102: 1083-1095, 2010.

17. Meadows AT, Friedman DL, Neglia JP, et al: Second neoplasms in survivors of childhood cancer: Findings from the Childhood Cancer Survivor Study cohort. J Clin Oncol 27: 2356-2362, 2009.

18. Varan A and Kebudi R: Secondary malignant neoplasms after childhood cancer. Pediatr Hematol Oncol 28: 345-353, 2011.

19. Danner-Koptik KE, Majhail NS, Brazauskas R, et al: Second malignancies after autologous hematopoietic cell transplantation in children. Bone Marrow Transplant 48: 363-368, 2013.

20. Faraci M, Bagnasco F, Corti P, et al: AIEOP-HSCT Group: Osteochondroma after hematopoietic stem cell transplantation in childhood. An Italian study on behalf of the AIEOP-HSCT group. Biol Blood Marrow Transplant 15: 1271-1276, 2009.

21. Garrison RC, Unni KK, McLeod RA, Pritchard DJ and Dahlin DC: Chondrosarcoma arising in osteochondroma. Cancer 49: 1890-1897, 1982.

22. Lamovec J, Spiler M and Jevtic V: Osteosarcoma arising in a solitary osteochondroma of the fibula. Arch Pathol Lab Med 123: 832-834, 1999.

23. Staals EL, Bacchini P, Mercuri M, and Bertoni F: Dedifferentiated chondrosarcomas arising in preexisting osteochondromas. J Bone Joint Surg Am 89: 987-993, 2007

24. Engel EE, Nogueira-Barbosa MH, Brassesco MS, et al: Osteosarcoma arising from osteochondroma of the tibia: Case report and cytogenetic findings. Genet Mol Res 11: 448-454, 2012.

25. Nojima T, Yamashiro K, Fujita M, et al: A case of osteosarcoma arising in a solitary osteochondroma. Acta Orthop Scand 62: 290-292, 1991.

26. Bovée JV, Sakkers RJ, Geirnaerdt MJ, Taminiau AH and Hogendoorn PC: Intermediate grade osteosarcoma and chondrosarcoma arising in an osteochondroma. A case report of a patient with hereditary multiple exostoses. J Clin Pathol 55: 226-229, 2002.

27. Meissner SA, Vieth V, August C, and Winkelmann W: Radiology-pathology conference: Osteosarcoma in a cartilaginous exostosis of the femur. Clin Imaging 30: 206-209, 2006. 